\title{
SELF-SIMILAR SOLUTIONS FOR THE KINEMATIC MODEL EQUATION OF SPIRAL WAVES
}

\author{
JONG-SHENQ GUO, NAOYUKI ISHIMURA, CHIN-CHIN WU
}

\begin{abstract}
We present a class of self-similar solutions of the kinematic model equation, introduced by V.A. Davydov, A.S. Mikhailov, and V.S. Zykov. This equation is designed to describe the dynamics of spiral waves in excitable media. In this model the sharply located spiral fronts are regarded as planar curves. If the tip neither grows nor retracts in the tangential direction and if their normal velocity (with the eikonal approximation) is assumed to possess no driving force, then the kinematic equation admits self-similar solutions with nonzero curvature. We show the global structure of both forward and backward self-similar solutions, which implies mathematically the existence of various types of spiral waves.
\end{abstract}

\section{INTRODUCTION}

Spiral waves are commonly observed in a variety of nonlinear sciences. We recall [7][13], for instance, rotating spiral waves in the Belousov-Zhabotinskii reagent, in cardiac arrhythmias, and in the aggregation of slime mold amoeba. These fascinating patterns are customary modeled by a sharp transition layer in the theory of reaction-diffusion systems on excitable media [4]. The system is typically referred to as "excitable" if a small stimulus is provoked to advance a long excursion and then returned to the resting state. Mathematically such character is incorporated into the singular perturbation analysis of the reaction-diffusion system, and in this picture, rotating spiral arms may be treated as traveling waves in excitable media [14].

On the other hand, ignoring the thickness of the interface and regarding it as a single curve, one may consider the motion of curves in order to explain theoretically such spiral pattern dynamics. Indeed, V.A. Davydov, A.S. Mikhailov, and V.S. Zykov introduced the following so-called kinematic model equation for curves (cf. [2][11][12] and $\S 2$ for the derivation):

$$
\kappa_{t}+v_{x x}+\left(\kappa \int_{0}^{x} \kappa v d \xi\right)_{x}+G \kappa_{x}=0, x>0, t>0
$$

where $x$ is the arc length parameter, $\kappa(x, t)$ is the curvature, $v(x, t)$ is the normal velocity, and $G:=G(t)$ is the tangential velocity of the tip (with $x=0$ ) for each time $t$.

If the eikonal approximation (i.e., $v=c-D \kappa$ ) is applied to the normal velocity and assume that the tip neither grows nor retracts in the tangential direction (i.e., $G(t) \equiv 0)$, then the equation (1.1) is reduced to

$$
\kappa_{t}-D \kappa_{x x}+\left(\kappa \int_{0}^{x} \kappa(c-D \kappa) d \xi\right)_{x}=0, x>0, t>0
$$

supplemented by the initial and boundary conditions. Physical constants $c$ and $D$ stand for the constant velocity of the solitary planar front and the diffusion coefficient of the trigger

Date: May 31, 2004. 
variable, respectively. For further information and other related topics, we refer additionally to [8][9][10] and the references cited therein.

In this paper, we shall confine ourselves to the case when $c=0$; that is, the normal velocity is assumed to contain no driving force term (see also [5]). By a re-scaling, we may also assume that $D=1$. Hence we shall study the equation

$$
\frac{\partial \kappa(x, t)}{\partial t}=\frac{\partial^{2} \kappa(x, t)}{\partial x^{2}}+\frac{\partial}{\partial x}\left(\kappa(x, t) \int_{0}^{x} \kappa(\xi, t)^{2} d \xi\right), x>0, t>0 .
$$

We are interested in seeking for some special exact nonzero solutions of (1.2). Since the equation (1.2) has the scaling invariance that if $\kappa(x, t)$ solves (1.2) then $\lambda \kappa\left(\lambda t, \lambda^{2} x\right)$ for $\lambda>0$ also does, it is expected that (1.2) admits self-similar type solutions. For example, $\kappa(x, t):=(T-2 t)^{-1 / 2}$ gives rise to a blowing-up self-similar solution (see Fig. 1 ), where $T$ is an arbitrarily given positive constant. Hereafter we assume that $\kappa$ is positive without loss of generality, since the equation (1.2) is invariant under the transformation $\kappa \rightarrow-\kappa$.

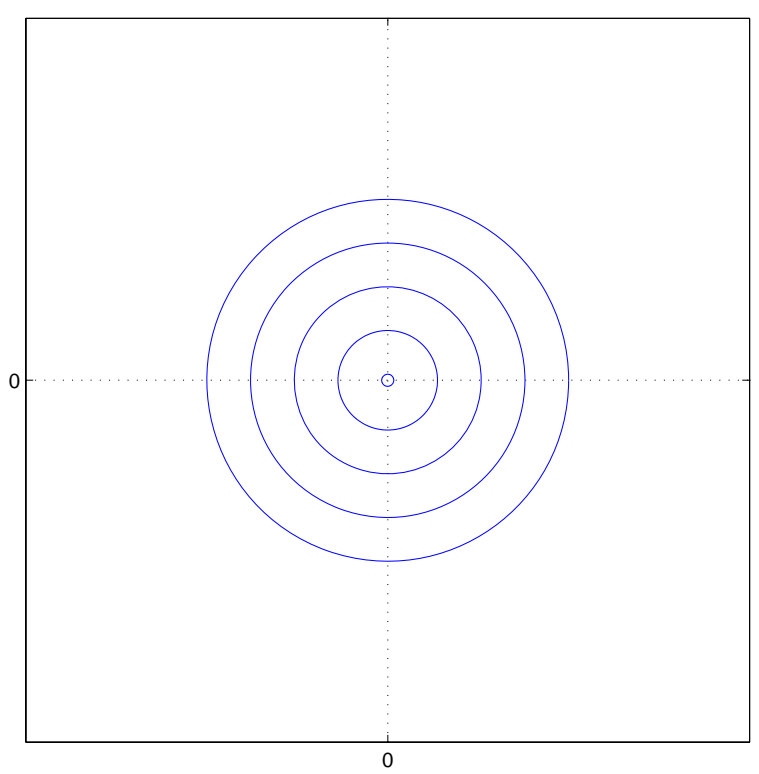

Figure 1. A self-similar blowing-up solution

We employ a usual self-similar change of variables. We put

$$
\begin{aligned}
& y=x(T-2 t)^{-1 / 2}, \quad \tau=-\frac{1}{2} \log (T-2 t), \\
& k(y, \tau)=(T-2 t)^{1 / 2} \kappa(x, t)
\end{aligned}
$$

for backward type and

$$
\begin{aligned}
& y=x(1+2 t)^{-1 / 2}, \quad \tau=\frac{1}{2} \log (1+2 t), \\
& k(y, \tau)=(1+2 t)^{1 / 2} \kappa(x, t)
\end{aligned}
$$

for forward type. We obtain that $k$ satisfies

$$
\frac{\partial k(y, \tau)}{\partial \tau}=\frac{\partial^{2} k(y, \tau)}{\partial y^{2}}+\frac{\partial}{\partial y}\left(k(y, \tau) \int_{0}^{y} k(\xi, t)^{2} d \xi\right) \mp \frac{\partial}{\partial y}(y k(y, \tau)), y>0
$$


where the - sign (resp., + sign) denotes the backward (resp., forward) self-similar version. The steady state of (1.7), namely, the solution with $\partial k / \partial \tau \equiv 0$ is called self-similar solution.

We want to classify all self-similar positive solutions of the kinematic equation (1.2). Precisely stated, we are seeking for positive global solutions (for $y \in[0, \infty)$ ) of the following ordinary differential equation

$$
k^{\prime \prime}(y)+\left(k(y) \int_{0}^{y} k(\xi)^{2} d \xi\right)^{\prime} \mp(y k(y))^{\prime}=0, y>0 .
$$

Then for any global solution $k$ of (1.8), the corresponding self-similar solution of (1.2) is given by

$$
\kappa(x, t):=(1+2 t)^{-1 / 2} k\left(x(1+2 t)^{-1 / 2}\right), x \geq 0, t \geq 0,
$$

for the forward case and

$$
\kappa(x, t):=(T-2 t)^{-1 / 2} k\left(x(T-2 t)^{-1 / 2}\right), x \geq 0,0 \leq t<T / 2,
$$

for the backward case, respectively. Our main results, which are principally mathematical nature, are formulated in Theorems 4.1, 4.2, 5.1, 5.2 and 5.7, according to the classification of equation types. Their implications to the real phenomena will be discussed in $\S 6$.

The study of nonlinear pattern formations often reveals that certain ubiquitous self-similar structures prevail in the phenomena. In terms of mathematical models, they are connected with the self-similar solutions, since characteristic behaviors of breakdown are asymptotically provided by these solutions, and therefore self-similar solutions form important classes of exact solutions [1]. In addition to the mathematical interest, we thus believe that the investigation of the structure of self-similar solutions to the kinematic model equation is worth publishing.

It turns out that our (1.8) is similar to the so-called steadily rotating spiral wave equation of (1.1), which is studied in [3][6], and makes it possible in part parallel treatments. However, they are indeed not the same equations nor present similar phenomena. The previous studies [3][6] are concerned with the case $\partial \kappa / \partial t \equiv 0$ in (1.2); namely, the steadily rotating spiral waves. While the present study is concerned with exact solutions of the full equation of (1.2); they are not steady state. Furthermore, from the mathematical viewpoint, the behavior of the nonlinear source terms is different between (1.8) and the one in [3][6]; we refer to the remark made just after (3.8) in $§ 3$.

This paper is organized as follows. In $\S 2$ we briefly review the derivation of the kinematic equation (1.1). Preliminaries as to the transformation of (1.8) are illustrated in $\S 3$. Then $\S 4$ is devoted to the analysis of the forward type equation and $\S 5$ to the backward type; the treatments are made along the similar lines of [3] with necessary modifications. We conclude the paper with discussions.

\section{Derivation of Equation (1.1)}

Here we quickly recall the derivation of the kinematic model equation (1.1). Although the equation is already treated in [2][11][12] for example, we make a brief review for the reader's convenience.

Let $\mathbf{z}=\mathbf{z}(x, t)$ denotes a family of curves in the plane evolving with time $t$, where $x$ is the arc length. Assume that these curves are moving with curvature flow: $v=v(\kappa)$, where $v$ is the normal velocity and $\kappa$ is the curvature. 
Recall that the normal velocity $v$ and the tangent vector $\mathbf{T}$ are given by

$$
v=\mathbf{z}_{t} \cdot \mathbf{n}, \quad \mathbf{T}=\mathbf{z}_{x},
$$

where the (left) normal vector $\mathbf{n}$ is defined by $\mathbf{n}:=i \mathbf{z}_{x}$. Recall also the Frenet-Serret Theorem in the plane that

$$
\left(\begin{array}{l}
\mathbf{T} \\
\mathbf{n}
\end{array}\right)_{x}=\left(\begin{array}{cc}
0 & -\kappa \\
\kappa & 0
\end{array}\right)\left(\begin{array}{l}
\mathbf{T} \\
\mathbf{n}
\end{array}\right)
$$

Then from $v_{x}=\mathbf{z}_{t x} \cdot \mathbf{n}+\mathbf{z}_{t} \cdot \mathbf{n}_{x}$ it follows that

$$
v_{x x}=\mathbf{z}_{t x x} \cdot \mathbf{n}+2 \mathbf{z}_{t x} \cdot \mathbf{n}_{x}+\mathbf{z}_{t} \cdot \mathbf{n}_{x x} .
$$

Since $\mathbf{z}_{x x}=\mathbf{T}_{x}=-\kappa \mathbf{n}$ and using $\mathbf{n} \cdot \mathbf{n}_{t}=0$, we have

$$
\mathbf{z}_{t x x} \cdot \mathbf{n}=(-\kappa \mathbf{n})_{t} \cdot \mathbf{n}=-\left(\kappa_{t} \mathbf{n}+\kappa \mathbf{n}_{t}\right) \cdot \mathbf{n}=-\kappa_{t} .
$$

Similarly, we have

$$
\mathbf{z}_{t x} \cdot \mathbf{n}_{x}=\mathbf{T}_{t} \cdot(\kappa \mathbf{T})=0
$$

and

$$
\begin{aligned}
\mathbf{z}_{t} \cdot \mathbf{n}_{x x} & =\mathbf{z}_{t} \cdot(\kappa \mathbf{T})_{x}=\mathbf{z}_{t} \cdot\left(\kappa_{x} \mathbf{T}+\kappa \mathbf{T}_{x}\right) \\
& =\left(\mathbf{z}_{t} \cdot \mathbf{T}\right) \kappa_{x}+\mathbf{z}_{t} \cdot\left(-\kappa^{2} \mathbf{n}\right)=\left(\mathbf{z}_{t} \cdot \mathbf{T}\right) \kappa_{x}-\kappa^{2} v
\end{aligned}
$$

Hence we deduce that

$$
v_{x x}=-\kappa_{t}-\kappa^{2} v+\left(\mathbf{z}_{t} \cdot \mathbf{T}\right) \kappa_{x} .
$$

On the other hand, we compute that

$$
\left(\mathbf{z}_{t} \cdot \mathbf{T}\right)_{x}=\mathbf{z}_{t x} \cdot \mathbf{T}+\mathbf{z}_{t} \cdot \mathbf{T}_{x}=\mathbf{T}_{t} \cdot \mathbf{T}+\mathbf{z}_{t} \cdot(-\kappa \mathbf{n})=-\kappa v .
$$

Therefore, we obtain that

$$
\left(\mathbf{z}_{t} \cdot \mathbf{T}\right)(x, t)=\left(\mathbf{z}_{t} \cdot \mathbf{T}\right)(0, t)-\int_{0}^{x} \kappa(\xi, t) v(\xi, t) d \xi .
$$

Now, we define the tangential velocity of the tip (denoted by $G$ ) by $G:=-\left(\mathbf{z}_{t} \cdot \mathbf{T}\right)(0, t)$. Then we obtain from (2.4) the following relation:

$$
\kappa_{t}+v_{x x}+\kappa^{2} v+\kappa_{x}\left(\int_{0}^{x} \kappa v d \xi\right)+G \kappa_{x}=0
$$

or equivalently

$$
\kappa_{t}+v_{x x}+\left(\kappa \int_{0}^{x} \kappa v d \xi\right)_{x}+G \kappa_{x}=0
$$




\section{Preliminaries}

In this section, we transform the nonlocal equation (1.8), namely,

$$
k^{\prime \prime}(y)+\left(k(y) \int_{0}^{y} k(\xi)^{2} d \xi\right)^{\prime} \mp(y k(y))^{\prime}=0
$$

to a local equation so that the standard ODE theory can be easily applied.

First, integration of (3.1) once yields

$$
k^{\prime}+k \int_{0}^{y} k^{2} d \xi \mp y k=-\eta,
$$

where $\eta:=-k^{\prime}(0)$. Multiply (3.1) by $k$ and (3.2) by $k^{\prime}$, respectively, we have

$$
\begin{aligned}
& k k^{\prime \prime}+k k^{\prime} \int_{0}^{y} k^{2} d \xi+k^{4} \mp k^{2} \mp y k k^{\prime}=0, \\
& \left(k^{\prime}\right)^{2}+k k^{\prime} \int_{0}^{y} k^{2} d \xi \mp y k k^{\prime}=-\eta k^{\prime},
\end{aligned}
$$

from where we deduce that

$$
k k^{\prime \prime}-\left(k^{\prime}\right)^{2}+k^{4} \mp k^{2}=\eta k^{\prime}
$$

after subtracting each other.

Now, by the assumption that $k$ is positive, the next change of variable is made:

$$
k(y)=e^{w(y)},
$$

which leads to

$$
w^{\prime \prime}+g_{\mp}(w)=\eta e^{-w} w^{\prime}, \quad w^{\prime}(0)=-\eta / \exp (w(0)),
$$

where $g_{\mp}(w):=e^{2 w} \mp 1$. We further define

$$
\begin{aligned}
E_{\mp}[w](y) & :=\frac{1}{2}\left(w^{\prime}(y)\right)^{2}+G_{\mp}(w(y)), \\
G_{\mp}(w) & :=\frac{1}{2} e^{2 w} \mp w .
\end{aligned}
$$

It is easy to verify that $G_{\mp}^{\prime}(w)=g_{\mp}(w)$ and

$$
\frac{d}{d y} E_{\mp}[w](y)=\eta e^{-w(y)}\left(w^{\prime}(y)\right)^{2} .
$$

We shall see that the sign of $\eta$ plays a crucial role in describing the profile of $w$.

We remark that the difference between the current cases and the work of [3], which will be useful in successive sections. Here the nonlinear source terms $g_{\mp}(w) \rightarrow \mp 1$ as $w \rightarrow-\infty$, while $g_{\text {prev }}(w):=e^{2 w}-a e^{w} \rightarrow 0$ as $w \rightarrow-\infty(a:=c / D)$ in [3][6]. Moreover the potential $G_{\mp}(w) \rightarrow \pm \infty$ as $w \rightarrow-\infty$, while $G_{\text {prev }}(w):=2^{-1} e^{2 w}-a e^{w} \rightarrow 0$ as $w \rightarrow-\infty$ (see Fig. 2).

Now the classification of self-similar solutions is reduced to that of the nonlinear oscillation (3.5). We shall denote the solution $w$ of $(3.5)$ with $w(0)=\ln b$ by $w(y ; b)$. Let $[0, R)$ be the maximal existence interval of $w$. Note that $R=R(b)$ and $R>0(R=\infty$ if $w$ is global $)$. Set

$$
\alpha:=\lim _{y \rightarrow R^{-}} E[w](y) .
$$

Then $\alpha$ always exists by virtue of (3.8). 


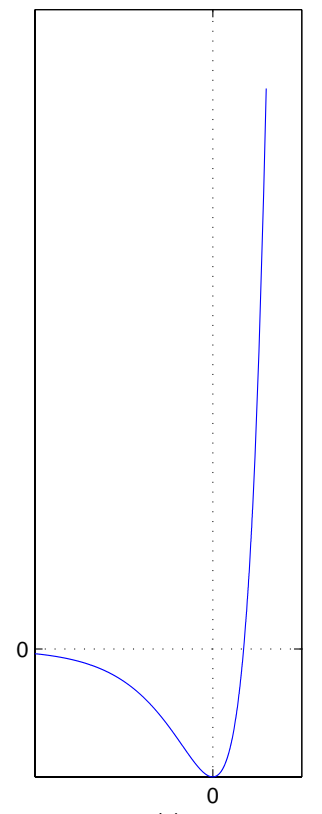

(a)

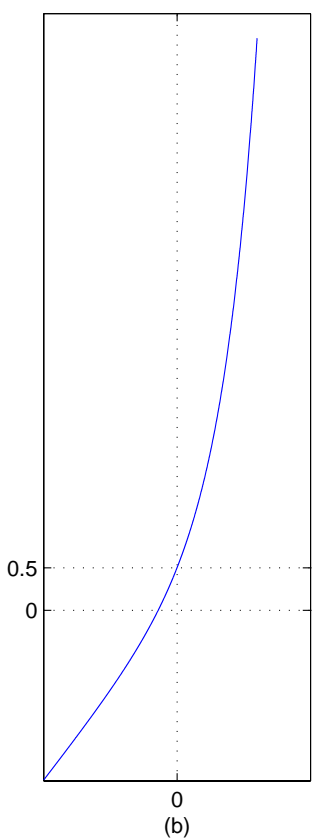

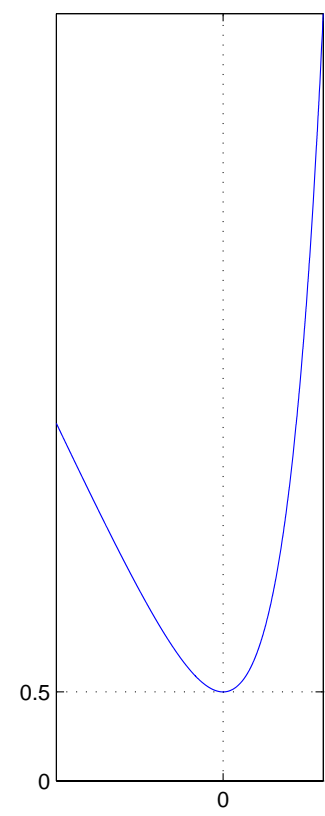

(c)

Figure 2. Graphs of potential functions for (a) $G_{\text {prev }}$; (b) $G_{+}$; and (c) $G_{-}$.

\section{FORWARD TYPE}

In this section, we deal with the forward type self-similar solution of (1.2), i.e., the plus sign in (3.5). Note that the function $G_{+}(w)=2^{-1} e^{2 w}+w$ is unbounded as $w \rightarrow \pm \infty$. Also, $g_{+}(w)=e^{2 w}+1>1$ for all $w$. Let $[0, R)$ be the maximal existence interval of $w$. We have the following result.

Theorem 4.1. Suppose $w=w(y)$ is a solution of

$$
w^{\prime \prime}+g_{+}(w)=\eta e^{-w} w^{\prime}, \quad w^{\prime}(0)=-\eta / \exp (w(0)),
$$

where $g_{+}(w)=e^{2 w}+1$. Then the following assertions hold.

(1) If $\eta<0$, then $w$ has exactly one critical point in $[0, R)$; that is, $w^{\prime}$ has exactly one zero in $[0, R)$. Moreover, $R=\infty$ and $w(y) \rightarrow-\infty$ as $y \rightarrow \infty$.

(2) If $\eta=0$, then $w=w(y)$ is globally monotone decreasing and $w(y) \sim-2^{-1} y^{2}$ as $y \rightarrow \infty$.

(3) If $\eta>0$, then $w$ is monotone decreasing to $-\infty$.

Proof. First, we deal with the case when $\eta<0$. Note that $w^{\prime}(0)>0$. Suppose for contradiction that $w^{\prime}>0$ in $[0, R)$. Then $R=\infty$. Otherwise, if $R<\infty$, then $w(y) \rightarrow \infty$ and $w^{\prime}(y) \rightarrow \infty$ as $y \rightarrow R^{-}$. This is impossible, since

$$
w^{\prime \prime}=\eta e^{-w} w^{\prime}-g_{+}(w)<-1 .
$$

We see that $R=\infty$. Set $l:=\lim _{y \rightarrow \infty} w(y)$ and $L:=\lim _{y \rightarrow \infty} w^{\prime}(y)$. Note that $l, L$ exist such that $l>-\infty$ and $L \in[0, \infty)$. If $l \in(-\infty, \infty)$, then $L=0$. This contradicts (4.2). On the other hand, if $l=\infty$, then $w^{\prime \prime}(y) \rightarrow-\infty$ as $y \rightarrow \infty$. This is also impossible, since we assume that $w^{\prime}>0$ in $[0, \infty)$. Notice that $w^{\prime \prime}<0$ if $w^{\prime}=0$. We conclude that $w$ has exactly one critical point $y_{0}$ in $[0, R)$ and $w^{\prime}<0$ in $\left(y_{0}, R\right)$. 
Suppose that $R<\infty$. Then it is clear that $w(y) \rightarrow-\infty$ as $y \rightarrow R^{-}$. Since $\alpha$ exists and $w^{\prime}<0$ in $\left(y_{0}, R\right)$, the limit $L:=\lim _{y \rightarrow R^{-}} w^{\prime}(y)$ exists and $L \leq 0$. If $L<0$, then it follows from (4.1) that $w^{\prime \prime}(y) \rightarrow \infty$ as $y \rightarrow R^{-}$, a contradiction. If $L=0$, then $w$ is bounded in $[0, R)$, a contradiction again. We conclude that $R=\infty$.

Set $l:=\lim _{y \rightarrow \infty} w(y)$. Then $l<\infty$. If $l>-\infty$, then there is a sequence $\left\{y_{n}\right\}$ such that

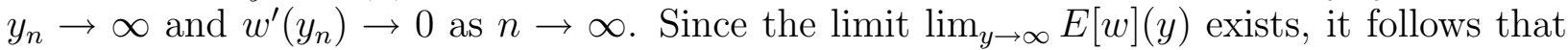
$L:=\lim _{y \rightarrow \infty} w^{\prime}(y)=0$. Then $w^{\prime \prime}(y) \rightarrow-g(l)$ as $y \rightarrow \infty$. This is a contradiction with $L=0$, since $-g(l)<-1$. Thus we obtain that $l=-\infty$.

Next, suppose that $\eta=0$. Then $w^{\prime \prime}=-g_{+}(w)<-1$. Hence $w^{\prime}<0$ in $(0, R)$, since $w^{\prime}(0)=0$. If $R<\infty$, then $w(y) \rightarrow-\infty$ as $y \rightarrow R^{-}$and so $w^{\prime \prime}(y) \rightarrow-1$ as $y \rightarrow R^{-}$. Thus $w^{\prime}$ is bounded in $[0, R)$, a contradiction. Therefore, $R=\infty$. It is trivial that $w(y), w^{\prime}(y) \rightarrow-\infty$ and $w^{\prime \prime}(y) \rightarrow-1$ as $y \rightarrow \infty$. This implies that $w(y) \sim-2^{-1} y^{2}$ as $y \rightarrow \infty$.

Finally, we study the case when $\eta>0$. Note that $w^{\prime}=0$ implies that $w^{\prime \prime}<0$. Hence $w^{\prime}<0$ in $[0, R)$. Since $w^{\prime \prime}<-1$ in $[0, R)$, it is easy to see that $w(y) \rightarrow-\infty$ as $y \rightarrow R^{-}$.

Now we want to determine whether $w$ is global or not for any $\eta>0$. Suppose that $w$ is a solution of (4.1) with $\eta>0$. Note that $w^{\prime}<0$ and $w^{\prime \prime}<-1$ in $[0, R)$. Hence the limit $L:=\lim _{y \rightarrow R^{-}} w^{\prime}(y)$ exists and $L<0(L$ may be $-\infty)$.

Theorem 4.2. Suppose that $\eta>0$. Then $R<\infty$.

Proof. For contradiction we suppose that $R=\infty$ for a solution $w$ of (4.1) with $\eta>0$. Note that from (4.1) it follows that $w^{\prime \prime}(y) \rightarrow-\infty$ as $y \rightarrow \infty$. Also, by differentiating the ode in (4.1) once, we can also derive that $w^{\prime \prime \prime}(y) \rightarrow-\infty$ as $y \rightarrow \infty$.

Applying the L'Hôpital's rule, we obtain that

$$
\begin{aligned}
\lim _{y \rightarrow \infty} y^{3} \exp [w(y)] & =\lim _{y \rightarrow \infty} \frac{y^{3}}{\exp [-w(y)]} \\
& =\lim _{y \rightarrow \infty} \frac{3 y^{2}}{-\exp [-w(y)] w^{\prime}(y)} \\
& =\lim _{y \rightarrow \infty} \frac{6 y}{-\exp [-w(y)] w^{\prime \prime}(y)+\exp [-w(y)]\left[w^{\prime}(y)\right]^{2}} \\
& =\lim _{y \rightarrow \infty} \frac{6}{-e^{-w(y)} w^{\prime \prime \prime}(y)+3 e^{-w(y)} w^{\prime}(y) w^{\prime \prime}(y)-e^{-w(y)}\left[w^{\prime}(y)\right]^{3}} \\
& =0 .
\end{aligned}
$$

Hence there is $y_{0}$ sufficiently large such that

$$
\exp [w(y)] \leq y^{-3}, \forall y \geq y_{0} .
$$

Now, from (4.1) it follows that

$$
w^{\prime}(y)+\eta e^{-w(y)}+\int_{y_{0}}^{y} g_{+}(w(s)) d s=A:=w^{\prime}\left(y_{0}\right)+\eta e^{-w\left(y_{0}\right)}
$$

and so

$$
e^{w(y)} w^{\prime}(y)+\eta+e^{w(y)} \int_{y_{0}}^{y} g_{+}(w(s)) d s=A e^{w(y)} .
$$

By an integration again we end up with

$$
e^{w(y)}-e^{w\left(y_{0}\right)}+\eta\left(y-y_{0}\right)+\int_{y_{0}}^{y}\left\{e^{w(\xi)} \int_{y_{0}}^{\xi} g_{+}(w(s)) d s\right\} d \xi=A \int_{y_{0}}^{y} e^{w(s)} d s .
$$


Note that $\left|g_{+}(w(s))\right| \leq 2$ for all $s \geq y_{0}$. Then it is easy to show that the integrals in (4.4) are uniformly bounded for all $y \geq y_{0}$. This leads a contradiction by letting $y \rightarrow \infty$. The proposition follows.

Hence we conclude that any solution $w$ of (4.1) is non-global for any $\eta>0$. In summary, the forward self-similar solutions of (1.2) exist if and only if $\eta \leq 0$. Note that $\kappa_{x}(0, t)=$ $-\eta(1+2 t)^{-1}$ and $\kappa(0, t)=\exp (w(0))(1+2 t)^{-1 / 2}$.

\section{BACKWARD TYPE}

In this section, a backward type, namely, the minus sign in (3.5), is handled. In this case, the function $G_{-}(w)=e^{2 w} / 2-w$ is bounded below by $1 / 2$. Let $w=w(y)$ be the solution of

$$
w^{\prime \prime}+g_{-}(w)=\eta e^{-w} w^{\prime}, \quad w^{\prime}(0)=-\eta / \exp (w(0))
$$

where $g_{-}(w)=e^{2 w}-1$.

5.1. The case for $\eta \leq 0$. First, for the case $\eta<0$, we have

Theorem 5.1. If $\eta<0$, then $w=w(y)$ enjoys the damped oscillation; precisely stated, $w$ is a global solution such that $w^{\prime}(y)$ changes sign infinitely many times and $w(y) \rightarrow 0$ exponentially as $y \rightarrow \infty$.

Next, for the case $\eta=0$, we obtain

Theorem 5.2. Let $\eta=0$. Then $w \equiv 0$ is the unique solution for (5.1) with $w(0)=0$. For $w(0) \neq 0$, the solution $w=w(y)$ is a periodic function with the period

$$
\sqrt{2} \int_{w^{-}}^{w^{+}} \frac{d w}{\sqrt{E[w](0)-G_{-}(w)}},
$$

where $w^{ \pm}\left(w^{+}>w^{-}\right)$satisfy $G_{-}\left(w^{ \pm}\right)=E[w](0)$.

These two theorems are easy to see; an elementary application of the theory of nonlinear oscillation suffices. See [3][6]. We may safely omit the proof. Note that the blowing-up self-similar solution $\kappa(x, t):=(T-2 t)^{-1 / 2}$ is corresponding to the case when $\eta=0$ and $w(0)=0$.

5.2. The case for $\eta>0$. For notational convenience, we shall drop the subscripts of $g$ and $G$. From now on, we set

$$
G(w)=\frac{1}{2} e^{2 w}-w, \quad g(w)=e^{2 w}-1 .
$$

We let $w=w(y)$ be the solution of the following initial value problem $(\mathrm{P})$ :

$$
\begin{aligned}
& w^{\prime \prime}+g(w)=\eta e^{-w} w^{\prime}, \\
& w(0)=\ln b, \quad w^{\prime}(0)=-\eta / b .
\end{aligned}
$$

We shall denote the solution by $w(y), w(y ; \eta), w(y ; \eta, b), w(y ; b)$ to specify the dependence of the parameter if it is necessary. As before, let $[0, R)$ be the maximal existence interval of $w$.

First, we have the following lemma.

Lemma 5.3. Let $\eta>0$. Suppose that the limit $l:=\lim _{y \rightarrow R^{-}} w(y)$ exists. Then $l=-\infty$. 
Proof. First, by an integration of (5.2) from 0 to $y$ we obtain the identity

$$
w^{\prime}(y)+\eta \exp \{-w(y)\}=-\int_{0}^{y} g(w(s)) d s .
$$

Suppose that $R=\infty$ and $l$ is finite. Then there is a sequence $\left\{y_{n}\right\}$ such that $y_{n} \rightarrow \infty$ and $w^{\prime}\left(y_{n}\right) \rightarrow 0$ as $n \rightarrow \infty$. Hence $w^{\prime}(y) \rightarrow 0$ as $y \rightarrow \infty$, since the limit $\alpha:=\lim _{y \rightarrow \infty} E[w](y)$ exists. Since $\alpha>E[w](0)>1 / 2$, we see that $l \neq 0$. This implies that $w$ is monotone ultimately. Hence there is a fixed sign for $g(w(s))$ for all $s$ sufficiently large and so the right-hand side of (5.4) tends to $+\infty$ or $-\infty$ as $y \rightarrow \infty$. But, the left-hand side of (5.4) remains bounded as $y \rightarrow \infty$, a contradiction. Thus we obtain that $l \in\{ \pm \infty\}$.

If $l=\infty$, then $w^{\prime}(y)>0$ for all $y$ sufficiently large and so the left-hand side of (5.4) is positive for all $y$ large enough. On the other hand, the right-hand side of (5.4) tends to $-\infty$ as $y \rightarrow \infty$. This is again a contradiction. Therefore, we conclude that $l=-\infty$.

Suppose that $R<\infty$. Then either $l=\infty$ or $l=-\infty$. Assume that $l=\infty$. Then $w^{\prime}(y) \rightarrow \infty$ as $y \rightarrow R^{-}$and so the left-hand side of (5.4) tends to $\infty$. On the other hand, by assumption there is $y_{0} \in(0, R)$ such that $w(y)>0$ for all $y \in\left(y_{0}, R\right)$. Then

$$
\begin{aligned}
-\int_{0}^{y} g(w(s)) d s & =-\int_{0}^{y_{0}} g(w(s)) d s-\int_{y_{0}}^{y} g(w(s)) d s \\
& <-\int_{0}^{y_{0}} g(w(s)) d s<\infty .
\end{aligned}
$$

This is a contradiction. Hence we must have $l=-\infty$.

Set $v=w^{\prime}$. Then the problem $(\mathrm{P})$ is equivalent to the following first order system $(\mathrm{Q})$ :

$$
\begin{aligned}
& w^{\prime}=v, \\
& v^{\prime}=\eta e^{-w} v-g(w)
\end{aligned}
$$

with the initial condition $(w, v)(0)=(\ln b,-\eta / b)$ for $b>0$.

We define the initial curve $\Gamma$ by

$$
\Gamma:=\left\{(w, v) \mid v=-\eta e^{-w}\right\} .
$$

Let $D_{1}$ and $D_{2}$ denote the region above and below the curve $\Gamma$, respectively. Then it is easy to see that the vector field on $\Gamma$ is pointed into $D_{2}$ if $w>0$ and is pointed into $D_{1}$ if $w<0$.

The nullcline of the system $(\mathrm{Q})$ is given by

$$
N:=\left\{(w, v) \mid v=f(w):=\left(e^{3 w}-e^{w}\right) / \eta\right\} .
$$

It is easy to see that $f(-\infty)=0, f^{\prime}(w)<0$ if $w<w_{0}, f^{\prime}(w)>0$ if $w>w_{0}$, where $w_{0}:=-\ln (\sqrt{3})$. Set $v_{0}:=f\left(w_{0}\right)=-2 /(3 \sqrt{3} \eta)$ and $c_{0}:=v_{0}^{2} / 2+G\left(w_{0}\right)$.

Recall that $E(w, v):=v^{2} / 2+G(w)$. The level set of $\Gamma_{c}:=\{E=c\}$ is nonempty if and only if $c \geq 1 / 2$. Moreover, for any $c>1 / 2$ the level set $\Gamma_{c}$ is a bounded closed curve, is symmetric with respect to the $w$-axis, and has two intersections with the $w$-axis (Fig. 3 ).

Let $\gamma:=\left\{(w, v) \mid w=w_{0}, v \leq v_{0}\right\}$ and let the region $\mathbb{A}$ is defined by the region below the nullcline $N$ and to the left of $\gamma$. Then we have the following positively invariance property for the region $\mathbb{A}$.

Lemma 5.4. The region $\mathbb{A}$ is positively invariant for the system (Q). 


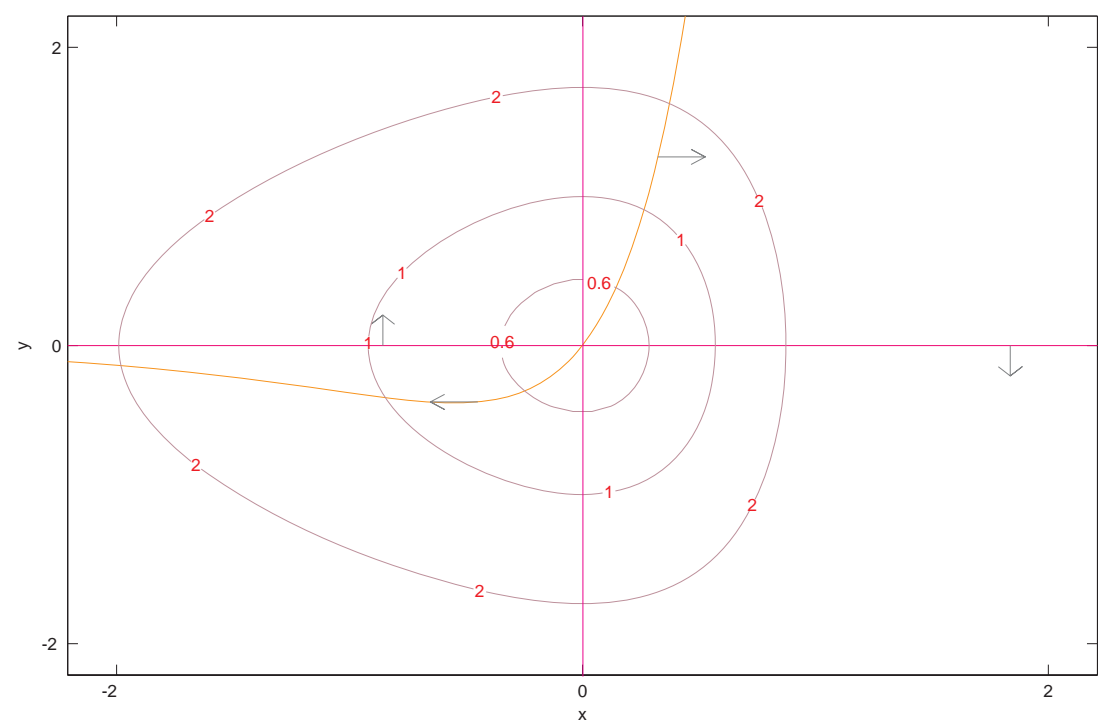

FiguRE 3. Nullcline and contours of energy level sets.

Proof. Since $w^{\prime}<0, v^{\prime}=0$ on $N \cap\left\{w \leq w_{0}\right\}$ and $w^{\prime}<0$ on $\gamma$, the region $\mathbb{A}$ is positively invariant.

We say that $w$ is oscillatory if $w^{\prime}$ has infinitely many zeros in $[0, R)$. Otherwise, we say that $w$ is non-oscillatory.

In the sequel, we shall consider solutions of (5.2) with more general initial data not just on $\Gamma=\left\{v=-\eta e^{-w}\right\}$ defined above. We should keep in mind that the solutions which are interested in are those solutions with initial data on $\Gamma$.

Lemma 5.5. Suppose that $\eta>0$. Then any solution $w$ of (5.2) is non-oscillatory. Hence $w(y) \rightarrow-\infty$ as $y \rightarrow R^{-}$for any solution $w$ of (5.1).

Proof. Suppose that there is a solution $w$ of (5.2) such that $w$ is oscillatory.

We first claim that $\alpha:=\lim _{y \rightarrow R^{-}} E[w](y)=\infty$. For contradiction, we assume that $\alpha<\infty$. Set $c:=\alpha$. Then $\Gamma_{c} \cap\{v=0\}=\{m, M\}$ for some constants $-\infty<m<M<\infty$. By assumption, there is a sequence $\left\{y_{n}\right\}$ with $y_{n} \rightarrow R^{-}$such that $w^{\prime}\left(y_{n}\right)=0$ for all $n$ and $w\left(y_{n}\right) \rightarrow M$ as $n \rightarrow \infty$, since $G\left(w\left(y_{n}\right)\right)=E[w]\left(y_{n}\right) \rightarrow \alpha$ as $n \rightarrow \infty$.

Now we consider the set

$$
I_{c}:=\left\{(d, 0) \mid E\left[w_{d}\right](y) \geq c \text { for some } y \geq 0\right\},
$$

where $w_{d}$ is the solution of $(5.2)$ with initial condition $w_{d}(0)=d, w_{d}^{\prime}(0)=0$. It follows from the theory of continuous dependence on initial data that the set $I_{c}$ is open. Here we used the fact that the energy is strictly increasing in $y$. Since $(M, 0) \in I_{c}$, there is a positive constant $\delta$ such that $(M-\delta, M+\delta) \times\{0\} \subset I_{c}$. Hence $\left(w\left(y_{n}\right), w^{\prime}\left(y_{n}\right)\right) \in I_{c}$ for all $n$ sufficiently large. This leads a contradiction, since the limit of the energy for any trajectory starting at $I_{c}$ must be strictly bigger than $c$. We conclude that $\alpha=\infty$. 
Next, since $w$ is oscillatory, there are sequences $\left\{y_{n}\right\}$ and $\left\{z_{n}\right\}$ with $y_{n}, z_{n} \rightarrow R^{-}$such that $w^{\prime}\left(y_{n}\right)=w^{\prime}\left(z_{n}\right)=0$ for all $n$ and $w\left(y_{n}\right) \rightarrow \infty$ and $w\left(z_{n}\right) \rightarrow-\infty$ as $n \rightarrow \infty$. Here we have used the fact that $G(w) \rightarrow \infty$ as $w \rightarrow \pm \infty$. From the above observation, we can also find a sequence $\left\{x_{n}\right\}$ tending to $R^{-}$such that $w\left(x_{n}\right)=w_{0}$ and $w^{\prime}\left(x_{n}\right)<0$ for all $n$. Since $\alpha=\infty$, we see that $w^{\prime}\left(x_{n}\right) \rightarrow-\infty$ as $n \rightarrow \infty$. Therefore, the trajectory of $w$ will reach the line $\gamma$ at $x_{n}$ for $n$ sufficiently large. Then this trajectory stays in the region $\mathbb{A}$ after $x_{n}$ for $n$ sufficiently large, by Lemma 5.4. This is a contradiction. Thus the lemma is proved.

We set $\tilde{L}:=\limsup _{y \rightarrow R^{-}} w^{\prime}(y)$. Note that $\tilde{L}$ always exists and $\tilde{L} \leq 0(\tilde{L}$ may be $-\infty)$, since $w^{\prime}(y)<0$ for all $y$ sufficiently close to $R$. We also remark that $\tilde{L}$ plays an equivalent role to the quantity $\lim _{y \rightarrow R^{-}} E(y)$ exploited in [3] (this quantity is denoted by $L$ there).

Proposition 5.6. Suppose that $\eta>0$ and $\tilde{L}<0$. Then $R<\infty$.

Proof. Suppose that there is a global solution $w$ with $\tilde{L}<0$. Then there is a positive constant $\sigma$ such that $w^{\prime}(y)<-\sigma$ for all $y$ sufficiently large. This implies that $w^{\prime \prime}(y), w^{\prime \prime \prime}(y) \rightarrow$ $-\infty$ as $y \rightarrow \infty$. Following the proof of Theorem 4.2, we reach a contradiction. Hence $R<\infty$. The proposition follows.

Conversely, if $R<\infty$, then $L$ (recall that $L:=\lim _{y \rightarrow R^{-}} w^{\prime}(y)$ ) exists and $L=-\infty$. To see this, we first observe that $g(w(y)) \rightarrow-1$ as $y \rightarrow R^{-}$. Hence the limit

$$
\lim _{y \rightarrow R^{-}} \int_{0}^{y} g(w(s)) d s
$$

exists and is finite, since $R<\infty$. Therefore, it follows from (5.4) and Lemma 5.5 that $L$ exists and $L=-\infty$. Consequently, $w$ is global if and only if $\tilde{L}=0$.

We conclude that there are only two different types solutions: one is global with $\tilde{L}=0$ and the other is non-global with $\tilde{L}<0$ (and so $L=-\infty$ ). We shall call the global one as a Type I solution; and the non-global one as a Type II solution. Note that $E[w](y) \rightarrow \infty$ as $y \rightarrow R^{-}$in any case.

5.3. Solution structure for $\eta>0$. To study the structure of solutions of (5.1) for a given $\eta>0$, we follow the method of [3] and divide our discussion into the following steps.

1. Consider for a fixed $\eta>0$ the problem (Q) with initial value $(0,-a)$ for $a>0$. Denote the corresponding solution by $w_{a}$. We prove that for each $\eta \in(0,1)$ there is a unique $\alpha=\alpha(\eta)>0$ such that $w_{a}$ is of Type I if and only if $a=\alpha$.

2. Vary $\eta$ and study the solution $w_{\eta}$ of the problem (Q) with initial value $(0,-\eta)$ for $\eta>0$. Determine $\eta_{0}$ so that (5.1) has a Type I solution if and only if $\eta \leq \eta_{0}$.

3. Define the sequence $\eta_{m}(m \geq 1)$, which distinguishes the multiplicity of Type I solutions to (5.1), inductively as in [3].

We now state the main theorem of this section as follows.

Theorem 5.7. There exists a strictly decreasing sequence $\left\{\eta_{m}\right\}$ with $\eta_{m} \rightarrow 0$ as $m \rightarrow \infty$ such that the problem (5.1) has exactly $2 m+1$ solutions of Type I, if $\eta=\eta_{m}$; has exactly $2 m$ solutions of Type I, if $\eta \in\left(\eta_{m}, \eta_{m-1}\right)$. All other solutions are of Type II.

To prove Theorem 5.7, we first observe that for a given $\eta>0$ the region

$$
\mathbb{B}:=\{(w, v) \mid w \leq 0, v \leq-1 / \eta\}
$$


is a positively invariant region for the system (Q). Then it is easily to see that $w$ is of Type II solution if and only if $\left(w(y), w^{\prime}(y)\right) \in \mathbb{B}$ for some $y \geq 0$. Hence the set $J_{1}$ is open, where

$$
J_{1}:=\left\{a>0 \mid w_{a}^{\prime}(y)<0 \text { for all } y \geq 0 \text { and } w_{a} \text { is of Type II }\right\} .
$$

Clearly, the set

$$
J_{2}:=\left\{a>0 \mid w_{a}^{\prime}(y)=0 \text { for some } y \geq 0\right\}
$$

is also open. If $\eta<1$, then $(0, \delta) \subset J_{2}$ for some $\delta>0$, since $(0,0)$ is a spiral point. Also, there holds $[1 / \eta, \infty) \subset J_{1}$ for any $\eta>0$. Then the number $\alpha=\alpha(\eta):=\inf J_{1}$ exists and is positive. Note that the corresponding solution $w_{\alpha}$ is strictly monotone decreasing. We claim that $w_{\alpha}$ is the unique (up to translations) Type I solution among all $w_{a}$ for all $a>0$. Indeed, if there is another $a \neq \alpha$ such that $w_{a}$ if of Type I, then it is clear that $a<\alpha$. For convenience, we set $w_{1}:=w_{\alpha}, w_{2}:=w_{a}, v_{i}:=w_{i}^{\prime}, i=1,2$. By a translation, we may assume that $v_{2}(y)<0$ for all $y \geq 0$ and $v_{2}(0)=-a>-\alpha$. Since we can view $v_{i}$ as a function of $w$, we have

$$
\frac{d v_{i}}{d w}=\eta e^{-w}+\frac{1-e^{2 w}}{v_{i}} \text { for } w \leq 0, i=1,2,
$$

and $v_{1}(0)=-\alpha, v_{2}(0)=-a$. Then $v_{1}^{\prime}(w)-v_{2}^{\prime}(w)>0$ for all $w<0$. By an integration from $w<0$ to 0 , we obtain that

$$
v_{1}(w)<v_{1}(w)-v_{2}(w)<v_{1}(0)-v_{2}(0)=a-\alpha<0
$$

for any $w<0$. Letting $w \rightarrow-\infty$ in (5.6), we reach a contradiction. Hence the uniqueness follows.

Next, we vary $\eta$ and denote the solution of $(\mathrm{Q})$ with initial value $(0,-\eta)$ by $w_{\eta}$ for $\eta>0$. Note that $w_{\eta}$ is of Type II, if $\eta \geq 1$ (so that $\eta \in[1 / \eta, \infty) \subset J_{1}$ ). We also note that the trajectory of $w_{\eta}$ stays in $\mathbb{B}$ for any $\eta \geq 1$. Consider the set

$$
A_{1}:=\left\{\eta>0 \mid w_{\eta}^{\prime}(y)=0 \text { for some } y>0\right\} .
$$

As in [3] we can show that $(0, \delta) \subset A_{1}$ for some small positive number $\delta$. On the other hand, since $A_{1}$ is bounded above by 1 , the number $\eta_{0}:=\sup A_{1}$ is well-defined and is positive. Then, by a comparison principle (cf. Lemma 4.9 in [3]), the solution $w_{\eta_{0}}$ is the unique monotone Type I solution among all $w_{\eta}$. Note that we also have $\eta_{0}=\inf A_{2}$, where

$$
A_{2}:=\left\{\eta>0 \mid w_{\eta}^{\prime}(y)<0 \text { for all } y \geq 0 \text { and } w_{\eta} \text { is of Type II }\right\} .
$$

Since two different trajectories of an autonomous system cannot intersect each other, a simple phase analysis gives that every solution of (5.1) is of Type II, if $\eta>\eta_{0}$. Conversely, a similar argument as of Theorem 1 in [3], we can show that there exists Type I solution for (5.1), if $\eta \leq \eta_{0}$. Indeed, if $w_{\eta}$ is of Type I, then we are done. Otherwise, $w_{\eta}$ is of Type II and the trajectory of $w_{\eta}$ only has finitely many intersections with $\gamma_{0}:=\{(0,-d) \mid d>0\}$, say, at $\left(0,-d_{i}\right), i=1, \ldots, N, N \geq 2$, with $\eta:=d_{1}<\cdots<d_{N}$. Then $d_{N-1} \in J_{2}$ and $d_{N} \in J_{1}$. Therefore, there exists the unique solution $w_{\alpha}$ of Type I with $\alpha \in\left(d_{N-1}, d_{N}\right)$. It is clear that the trajectory of $w_{\alpha}$ intersects the initial curve. This proves the existence of Type I solution for (5.1), if $\eta \leq \eta_{0}$.

Finally, we define the sequence $\left\{\eta_{m}\right\}$ inductively by $\eta_{m}:=\inf B_{m}$, where

$$
B_{m}:=\left\{\eta<\eta_{m-1} \mid w_{\eta} \text { has exactly } 2 m+1 \text { zeros and is of Type II }\right\}
$$


for $m \geq 1$. To begin with the proof of Theorem 5.7, we consider the level curve $\Gamma_{c}$ with $c=\eta_{0}^{2} / 2+1 / 2$. Let $\left(w_{0}, 0\right)$ be the unique point on $\Gamma_{c}$ with $w_{0}<0$. Note that there is a one-to-one correspondence of the trajectory connecting the segment $\left\{(0,-d) \mid 0<d<\eta_{0}\right\}$ and the half-line $\{(w, 0) \mid w \leq 0\}$. Therefore, there exists an $\hat{\eta} \in\left(0, \eta_{0}\right)$ such that the trajectory from $(0,-\hat{\eta})$ will be connected to $\left(w_{0}, 0\right)$. By a simple phase plane analysis, $w_{\eta}$ has exactly 3 zeros and is of Type II for any $\eta \in\left(\hat{\eta}, \eta_{0}\right)$. This proves that the set $B_{1}$ is non-empty. It is clear that $B_{1}$ is bounded below, since the smaller $\eta$ is the more oscillations $w_{\eta}$ has. This implies that $\eta_{1}$ is well-defined and the corresponding trajectory has exactly 3 zeros and is of Type I.

The rest of the proof is similar to that of Theorem 2 in [3] and we safely omit it here.

In summary, there are infinitely many backward self-similar solutions of (1.2) for $\eta \leq 0$. For $\eta>0$, there is a positive constant $\eta_{0}$ such that a backward self-similar solution of $(1.2)$ exists if and only if $\eta \in\left(0, \eta_{0}\right]$. Moreover, the number of backward self-similar solutions of (1.2) can be counted exactly for each $\eta \in\left(0, \eta_{0}\right]$. Note that $\kappa_{x}(0, t)=-\eta(T-2 t)^{-1}$ and $\kappa(0, t)=\exp (w(0))(T-2 t)^{-1 / 2}$.

\section{Discussions}

We have investigated the structure of self-similar solutions of the kinematic equation, which is introduced to model the dynamics of steadily rotating spiral waves in excitable media. Selfsimilar solutions are known to exhibit a prototype behavior in various nonlinear sciences. Under somewhat restrictive assumptions that there is no driving force in the normal velocity (with the eikonal approximation) and the tip neither grows nor retracts in the tangential direction, this kinematic equation has a family of self-similar solutions. Both forward and backward types of self-similar equations admit solutions which realize spiral-shaped curves.

We give some explanations for the mathematical results which we obtained in Sections 4 and 5. In Section 4, we deal with the forward self-similar solutions in the form

$$
\kappa(x, t):=(1+2 t)^{-1 / 2} \exp \left\{w\left(x(1+2 t)^{-1 / 2}\right)\right\}, x \geq 0, t \geq 0 .
$$

Recall that any solution of (4.1) is bounded above. Hence, as $t \rightarrow \infty$, we have $\kappa(x, t) \rightarrow 0$ for any $x \geq 0$. This means that the corresponding curves become flattening as $t$ increases and eventually become a flat half-line. Hence Theorem 4.1 gives us the existence of self-similarly flattening solutions.

On the other hand, in Section 5, the self-similarly blowing-up solutions in the form

$$
\kappa(x, t):=(T-2 t)^{-1 / 2} \exp \left\{w\left(x(T-2 t)^{-1 / 2}\right)\right\}, x \geq 0,0 \leq t<T / 2,
$$

are obtained in Theorems 5.1, 5.2, 5.7. If we consider the curve for any specific time as a snap-shot of camera, then the evolution of a backward self-similar solution can be seen as a zoom-out process. Hence the blowing-up of curvature is corresponding to the shrinking of the curves to a point.

Their whole structures, however, are complex enough and the relation with the physical phenomena seems need further examinations. We point out several difficulties in interpreting our results as real phenomena.

In many cases, both physically and chemically relevant spirals are Archimedean. We recall that the Archimedean spiral is defined as a curve whose radius from the center is proportional to the angle variable. This kind of spirals has a constant pitch, which reflects a periodic movement of the reaction-diffusion system. In our previous paper [6] on steadily 
rotating spiral waves, a stress is placed on the existence of asymptotically Archimedean spirals in the sense that $\lim _{x \rightarrow \infty} \kappa(x)^{2} x=$ constant. Here the current paper is concerned with the self-similarly moving solution and the Archimedean property is not so clear.

Conversely, from the practical viewpoint, it is desirable to ask whether there exist occasions in real world, which correspond to the behavior of self-similar spirals we proved, even if approximately. This may be an experimentally interesting issue for research. Moreover, the structure for the backward type with $\eta>0$ happens to be similar as the one for the steadily rotating spiral waves with $\eta>0$ established in [3]. It may be also challenging to find out what mechanism brings this coincidence.

Nevertheless we believe that our analysis, principally based on the mathematical standpoint, may shed light on the validity and/or the limitation of the kinematic model equation.

\section{Acknowledgements.}

We are grateful to anonymous referees for precious suggestions, which helps improving the manuscript a lot. This work was partially supported by the National Science Council of the Republic of China under the grant NSC 92-2115-M-003-008. Part of this work was done during a visit of the first author (J.-S. Guo) to Hokkaido University and Nihon University, Japan, in 2004. He would like to thank these two institutes for the hospitality during his visit.

\section{REFERENCES}

[1] G.I. Barenblatt; Scaling, Self-similarity, and Intermediate Asymptotics, Graduate Texts in Appl. Math., 14, Cambridge Univ. Press., 1996.

[2] V.A. Davydov, V.S. Zykov, \& A.S. Mikhailov; Kinematics of autowaves structure in excitable media (English translation), Soviet Phys. Usp., 34 (1991), 665-684.

[3] B. Fiedler, J.-S. Guo, \& J.-C. Tsai; Multiplicity of rotating spirals under curvature flows with normal tip motion, J. Diff. Equations (to appear).

[4] P.C. Fife; Dynamics of Internal Layers in Diffusive Interfaces, CBMS-NSF Regional Conference Series in Appl. Math., 53, 1988.

[5] Y. Giga, N. Ishimura, \& Y. Kohsaka; Spiral solutions for a weakly anisotropic curvature flow equation, Adv. Math. Sci. Appl., 12 (2002), 393-408.

[6] R. Ikota, N. Ishimura, \& T. Yamaguchi; On the structure of steady solutions for the kinematic model of spiral waves in excitable media, Japan J. Indust. Appl. Math., 15 (1998), 317-330.

[7] W. Jahnke \& A.T. Winfree; A survey of spiral-wave behaviors in the Oregonator model, Int. J. Bifur. Chaos, Appl. Sci. Eng., 1 (1991), 445-466.

[8] J.P. Keener; The core of the spiral, SIAM J. Appl. Math., 52 (1992), 1370-1390.

[9] J.P. Keener \& J.J. Tyson; The dynamics of scroll waves in excitable media, SIAM Rev., 34 (1992), $1-39$.

[10] E. Meron; Pattern formation in excitable media, Physics Reports, 218 (1992), 1-66.

[11] A.S. Mikhailov, V.A. Davydov, \& A.S. Zykov; Complex dynamics of spiral waves and motion of curves, Physica D, 70 (1994), 1-39.

[12] A.S. Mikhailov \& V.S. Zykov; Kinematical theory of spiral waves in excitable media: comparison with numerical computation, Physica D, 52 (1991), 379-397.

[13] J.D. Murray; Mathematical Biology, I: An Introduction, II: Spatial Models and Biomedical Applications, 3rd edition, Interdisciplinary Appl. Math., Springer, 2002, 2003.

[14] J.J. Tyson \& J.P. Keener; Singular perturbation theory of traveling waves in excitable media (A Review), Physica D, 32 (1988), 327-361.

Department of Mathematics, National Taiwan Normal University, Taipei 116, Taiwan.

E-mail address: jsguo@math.ntnu.edu.tw 
Department of Mathematics, Graduate School of Economics, Hitotsubashi University, KUNITACHI, TOKYO 186-8601, JAPAN.

E-mail address: ishimura@math.hit-u.ac.jp

Department of Mathematics, National Taiwan Normal University, Taipei 116, Taiwan.

E-mail address: chin@hp715.math.ntnu.edu.tw 